\title{
NuMERICAL INVESTIGATION OF THE HEMOSTASIS PROCESS IN TRANSIENT BLOOD FLOW
}

\section{Gábor Závodszky, György Paál}

Budapest University of Technology and Economics, Department of Hydrodynamic Systems gzavodszky@gmail.com

\begin{abstract}
Thrombus formation is a thoroughly researched area, posing several unanswered questions. Quite many of the underlying processes are still not well understood. This uncertainty arises from the fact that the blood clotting mechanism in our bodies involves a rather complex reaction cascade with plenty of components. These underlying dynamic processes stretch over the domains of several disciplines (as there are biomechanical and biochemical reactions as well as fluid dynamical components). In this study the factors thought to be the most influential were selected and coupled with the transient flow field that reacts not only to the cardiac pressure waves but also to the changing geometry of the vessel due to the clot formation. Although the number of degrees of freedom is reduced heavily, this model is already capable of qualitatively reproducing the results of in-vivo measured hemostasis.
\end{abstract}

Keywords: coagulation, platelets, computational fluid dynamics

\section{Introduction}

The cascade process of the hemostasis is a vital self-defense process exhibited by our body. This is a natural response of the cardiovascular system that prevents undesirable blood loss in case of vascular injury. The blood coagulation is an important step during the hemostasis and its highly complicated cascade process is driven by several factors that can act either as a promoter or an inhibitor. The main steps include the aggregation and the binding of the platelets that are dependent on the local flow properties amongst other circumstances. Simulating this aggregation phenomenon is a challenging task as one has to make several appropriately chosen approximations. Anand et al. reviewed ${ }^{1}$ some of the interacting mechanisms regarding clot formation in flowing blood, and even though they deal with a narrower collection of participating processes than it is known in the current literature, from the view of a numerical simulation they are still numerous. Nesbitt et al. provided $^{2}$ some more insight into the thrombus formation by emphasizing the strong coupling between the biochemical processes and the local hemodynamic properties. According to their experimental work, platelet aggregation is influenced by emerging shear forces as platelet adhesion favours low-shear zones. Several other authors recorded the ongoing process of thrombus formation: for example Celi et al. recorded ${ }^{3}$ the thrombus formation in the microcirculation of a living mouse using widefield video-microscopy. Similar measurements were carried out using different reagents to highlight the concentration of the main components of the formation., ${ }^{4,5}$ While the number of high quality recording of this phenomena is increasing, the numerical simulations are still in a rather early stage. There are several pieces of work aiming at simulating thrombus formation numerically, but most of them cover only portions of the whole process. ${ }^{6-9}$ They try to solve the complex problem by approaching it either from the biochemical side and com- 
pute the reactions and the densities of the reagents, but fail to couple these equations with the transient (time-varying) flow field, or from the kinetic side, which usually lacks the appropriate handling of platelet concentrations. The platelet concentration profile inside the blood vessels is an important factor, which is far from being constant in either venous or arterial vessels. ${ }^{10}$ A lot of numerical models have been developed in the past decade in order to calculate the platelet concentration profile. ${ }^{11-13}$ Their main findings are that the marginating property of platelets is mainly caused by the finite size effect of both the smaller platelets and the significantly larger red blood cells (RBC). Technically the rolling motion of the larger RBCs pushes the platelets to the sides of the flow channel, thus creating a highly uneven platelet distribution across the diameter of the vessels. This drift force that acts upon platelets in blood flow seems to be proportional to the average local flow velocity. To prove this interaction between platelets and RBCs, Mountrakis et al. simulated ${ }^{14}$ particle collisions in blood flow using the immersed boundary method.

\section{Method}

During our simulations the blood coagulation mechanism made the geometry change in time. For this reason a two-dimensional lattice Boltzmann based fluid solver was implemented on a typical D2Q9 lattice to carry out the computations. This fluid solver method has a clear advantage in handling complex and changing geometries over the more conventional finite-volume solvers. The theoretical bases of this method were laid down by Bhatnagar, Gross and Krook (BGK), ${ }^{15}$ and were later developed to the level of general usability for fluid flows by Quian et al. ${ }^{16}$ We employed the so-called multiple-relaxation-time lattice Boltzmann model, ${ }^{17}$ which, for our case pro- vided increased numerical stability compared to the widely used BGK model.

The platelets immersed in the blood flow were simulated as a passive scalar concentration field. The passive attribute means that although the platelet distribution is driven by the pulsating blood flow, it has no influence back on it. This approximation is supported by the fact that the usual volumetric ratio of the platelets in blood is under $1 \%$. The advection-diffusion of these platelets was handled by the LBM method in the following way: when the velocity is prescribed by an already given velocity field (like in this case by the blood flow), the equilibrium function (from the original function ${ }^{16}$ ) of the scalar field reduces to the following:

$f_{i}^{e q}=\omega \rho\left(1+3 \overrightarrow{e_{l}} \cdot \vec{u}\right)$

where $\omega$ is the relaxation frequency, $\overrightarrow{e_{l}}$ is the $i$-th discretized velocity direction, $\vec{u}$ is the local velocity of the blood flow, and is the platelet concentration that can be computed analogously to fluid density:

$$
\rho=\sum_{i=1}^{9} f_{i}
$$

where $f_{i}$ is the particle distribution along the $i$-th discretized velocity on the lattice. The diffusion coefficient $D$ then analogous to the numerical viscosity:

$D=\frac{1}{3}\left(\tau-\frac{1}{2}\right)$

where $\tau=\frac{1}{\omega}$ is the relaxation time for the equilibrium function.

To acquire a proper platelet density profile, the interaction with the RBCs must be taken into account. The platelet drifting is mainly caused by the rolling motion of the RBCs, which is in turn caused by the emerging shear forces in the fluid. To simulate this marginating effect, a virtual force $\overrightarrow{F_{M}}$ has been prescribed that acts 
upon the density of the platelets. This force actually accounts for the finite size effects of the platelets and RBCs. The drift force acting on real platelets always drives platelets out from the main flow, to the sides of the vessel. It is a required behavior, as this causes increased platelet concentration in the vicinity of vessel walls where any injury can possibly occur. This $\overrightarrow{F_{M}}$ force points to the direction of the local largest shear stress decrease (in other words, to the direction of the smallest, most negative shear stress gradient). Its magnitude is proportional to the magnitude of the shear stress emerging in this specified direction. (We note here that this proportionality ratio will require a proper parameter study later. In the current work, it takes the value of unity.) Using this drift force, two simple channel flows were simulated with Reynolds numbers that are typical in these smaller vessels. The vessel wall was taken as a smooth, no-slip, rigid wall. The results of the simulations were compared to the experimental results of Woldhuis et al. ${ }^{16}$

The first simple channel flow simulation used constant velocity inlet and constant pressure outlet boundaries. The Reynolds number was set to one that is a typical value in venules. The result of the simulation is summarized below in Figure 1.

The platelet concentration shows a good qualitative agreement with the experimental results. This numerical model was tested with pulsatile flow as well, in order to reinforce the statement of its validity. For this reason, the other simulation used a time-varying inlet velocity boundary condition. Figure 2 shows the inlet flow rate profile that mimics the behaviour of a real cardiac pressure wave.

The Reynolds number defined with the maximum of the possible inlet velocity was set to ten, which is a usual value for arterioles. The results of the simulation were also compared

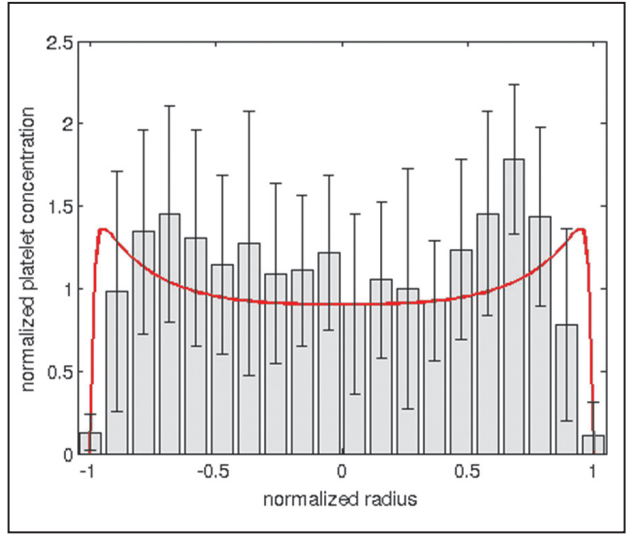

Figure 1. Normalized platelet concentration profile in a venule. The continuous red curve shows the results of the stationary simulation plotted over the experimental results of Woldhuis et al. ${ }^{18}$ $(\operatorname{Re}=1)$

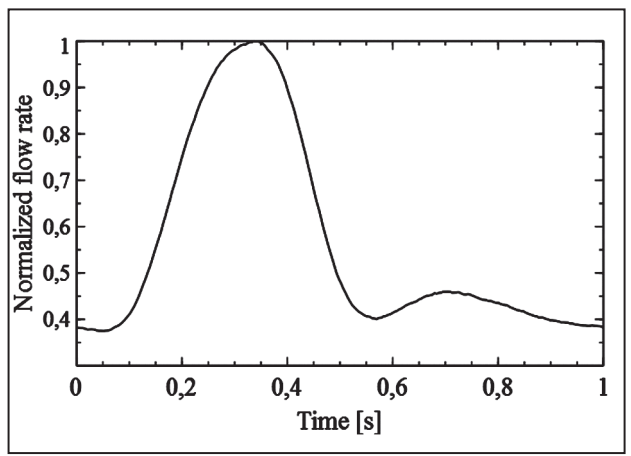

Figure 2. Normalized artificial flow rate curve that mimics the behaviour of a real flow rate curve during a cardiac cycle

to the experimental results of the same authors in Figure 3. Again, good qualitative agreement was found. The biggest deviation between the simulation and experimental results can be found very close to the vessel walls but it is still within the error bars.

The coagulation of blood is modeled by changing the type of a numerical element from fluid cell to solid cell. For the thrombus formation in our simulation, coagulation is only possible at sites right next to vessel walls or next to rigid 


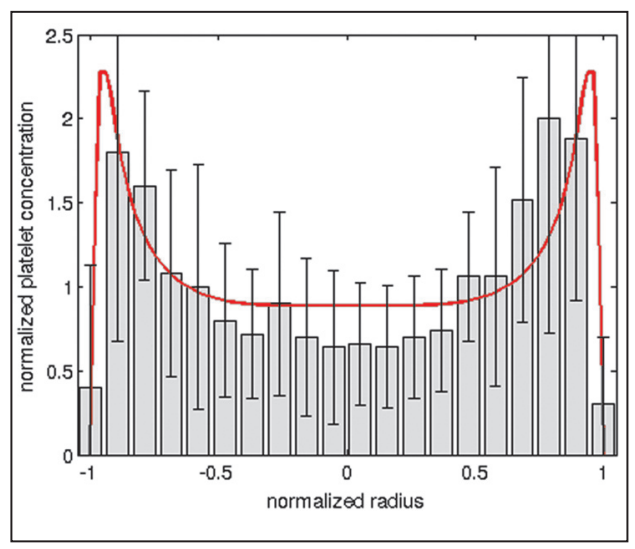

Figure 3. Time-averaged normalized platelet concentration profile in an arteriole.

The continuous red curve shows the results of a transient simulation plotted over the experimental results of Woldhuis et al. ${ }^{18}(\mathrm{Re}=10)$

blood cells that have already come to stasis. All the fluid LBM cells next to a wall cell were tracked, as these cells have the ability to turn into a solid cell themselves. When this happens, their fluid cell neighbours are added to the list of near-wall cells. Though platelet activation in reality is a complex cascade process, we only simulate one reagent, the adenosine diphosphate (ADP) concentration that is in our simulation not coupled with the blood flow. This means that the ADP concentration is not influenced by the local flow properties directly. When a platelet is activated (or in our simulation when coagulation of a numerical lattice happens), it releases ADP that can initiate the activation of other platelets. In the simulations the ADP concentration decreases exponentially in space, so its release can be thought of as a local ADP concentration increase.

Coagulation at a numeric lattice site is dependent on the relation of three variables:

$$
P_{\text {coag }}=\frac{\rho_{\text {platelet }} \cdot \rho_{A D P}}{\tau_{M A X}}
$$

where the $P_{\text {coag }}$ is a probability in the sense, that its value decides whether a fluid cell should come to stasis or not at any given time, based on the current local platelet concentration $\rho_{\text {platelet, }}$ the local ADP concentration $\rho_{A D P}$, and the local maximum shear component of the stress tensor denoted by $\tau_{M A X}$. The threshold level of $P_{\text {coag }}$ that a numerical lattice has to reach for coagulation is an empirical parameter of the model for now. A later work should explore the deeper relation of these parameters. For a numerical fluid cell to come to stasis the parameters have to remain in the coagulation zone for a $t_{\text {window }}$ time. This timewindow was chosen to be $t_{\text {window }}=20 \mathrm{~ms}$ because this is the usual timeframe for ADP to activate a platelet. This also means that a newly registered near-wall lattice cannot turn into a solid cell sooner than $20 \mathrm{~ms}$.

\section{Results}

In Figure 4 a visualized snapshot of a coagulation simulation is shown after six heartbeat cycles. The formation of the thrombus starts at an injury site. In in-vivo systems in case of vascular injury, a significant amount of $\mathrm{ADP}$ is released into the bloodstream from the injured vessel wall tissue. The injury site in this case was simulated by increasing the ADP concentration at a small amount of numerical lattices along the vessel wall. There are a few qualitative features of the calculated thrombus that should be mentioned. Firstly, as the thrombus begins to block the flow channel, the velocity increases and that leads to larger emerging shear stresses that inhibit the process of the coagulation. This results in the relatively flat top of the thrombus. It is also noticeable that the thrombus growth is asymmetric to the site of injury; its growth is slower on the side facing the incoming bloodstream. This is again the result of the different shear stresses.

The simulation results were compared to two thrombus geometries recorded at different times with video-microscopy by Nesbitt et al. ${ }^{10}$ 

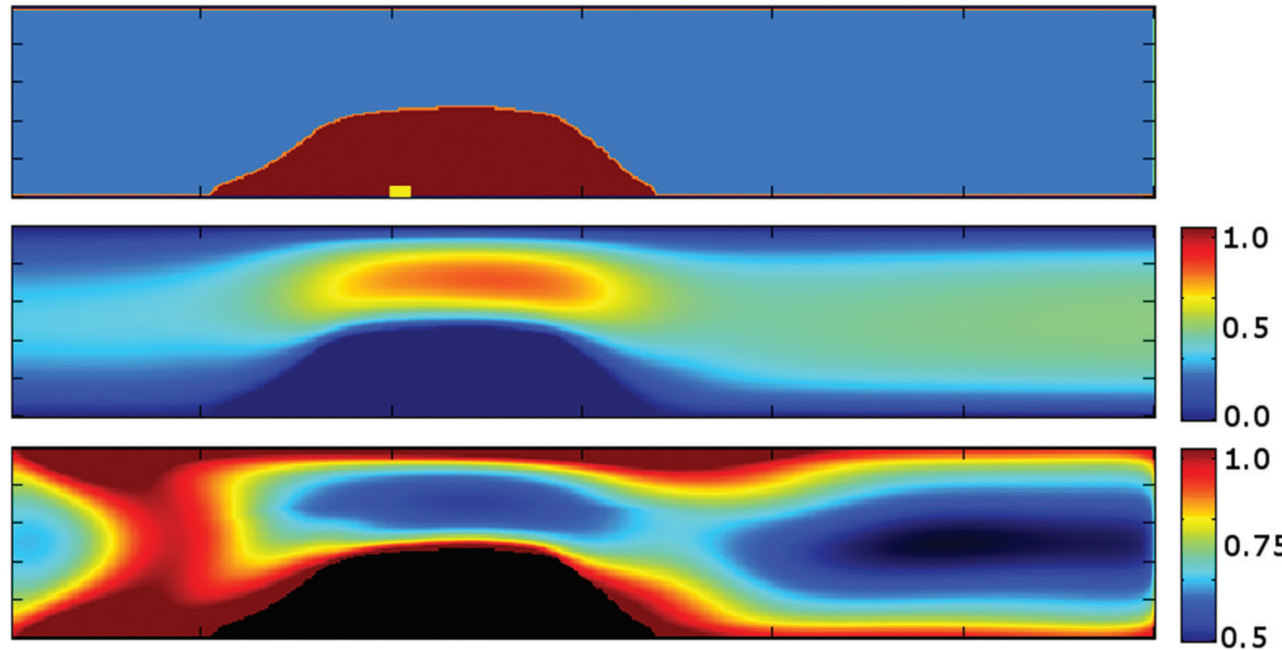

Figure 4. Typical result of a thrombus formation simulation at time instant of the end of a heartbeat cycle.

The flow direction is from the left to the right. The upper image shows the emerged geometry of the thrombus, with the place of vessel injury (yellow box at the bases of the thrombus).

The middle image shows the current normalized velocity magnitudes (Reynolds number equals 10).

The lower image shows the normalized platelet concentration at the same instant

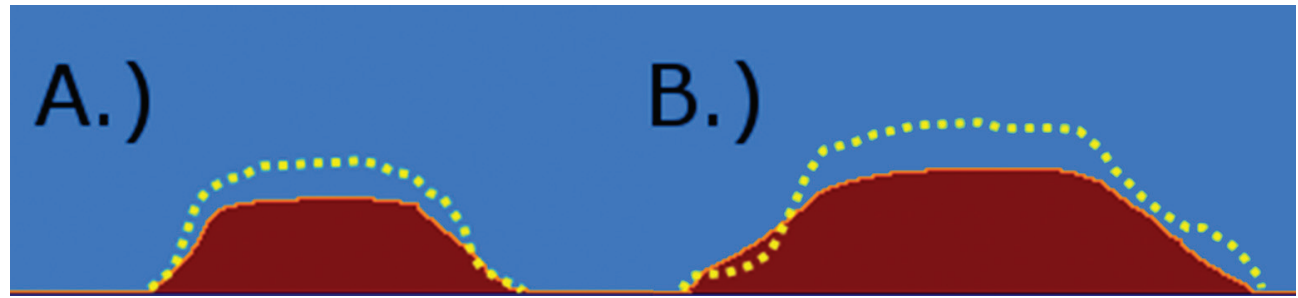

Figure 5. Geometry of the thrombus after three A.) and after six B.) heart beat cycles. The yellow dotted outline shows the geometry of the thrombus recorded with video-microscopy by Nesbitt et al. ${ }^{2}$

in Figure 5. The experimental results originate from an induced injury inside the arteriole of a living mouse. The exact form of the thrombus naturally depends on much more components that are taken into account in the current numerical simulation. Still, some qualitative features like effects of shear stresses can be studied with it.

\section{Conclusions}

Hemostasis is a heavily studied topic that represents deep complexity. The aim of a numeri- cal simulation is to reproduce certain features of a phenomena using the smallest necessary state space. In this work a numerical model was presented for hemostasis that incorporates different contributing disciplines in a computationally effective way. And though this model evidently has some shortcomings, like that in a real thrombus the outer layer is often not solid, and can dissolve easily in the flow and subside again later. In our model, however, the thrombus is handled as solid material, unable to dissolve. The effects of the several other components of the coagulation cascade were 
not taken into account either. Still, according to the comparison these key components coupled with the blood flow seems to be sufficient to reproduce the major characteristics of an injury induced thrombus. It should also be mentioned that due to the strongly simplified nature of the model, it might be capable of simulating other sediments in blood flows, like the deposition of fat particles on clinical devices.

\section{REFERENCES}

1. Anand M, Rajagopal KR. A model incorporating some of the mechanical and biochemical factors underlying clot formation and dissolution in flowing blood. Journal of Theoretical Medicine 2003;5:183-218.

2. Nesbitt WS, Westein E, Tovar-Lopez FJ, Tolouei E, Mitchell A, Fu J, Carberry J, Fouras A, Jackson $S P$. A shear gradient-dependent platelet aggregation mechanism drives thrombus formation. Nature Medicine 2009;15:665-73.

3. Celi A, Merrill-Skoloff G, Gross P, Falati S, Sim $D S$, Flaumenhaft R, Furie BC, Furie B. Thrombus formation: direct real-time observation and digital analysis of thrombus assembly in a living mouse by confocal and widefield intravital microscopy. Journal of thrombosis and haemostasis 2003;1(1):60-8.

4. Falati S, Gross P, Merrill-Skoloff G, Furie BC, Furie B. Real-time in vivo imaging of platelets, tissue factor and fibrin during arterial thrombus formation in the mouse. Nature Medicine 2002;8:1175-81.

5. Furie $B$, Furie $B C$. In vivo thrombus formation. Journal of thrombosis and haemostasis 2007; $5(1): 12-7$.

\section{Alenitsyn AG, Kondratyev AS, Mikhailova I, Sid-} dique I. Mathematical modeling of thrombus growth in microvessels. Journal of Prime Research in Mathematics 2008;4:195-205.

\section{Harrison SE, Smith SM, Bernsdorf J, Hose DR,} Lawford PV. Application and validation of the lattice Boltzmann method for modelling flowrelated clotting. Journal of Biomechanics 2007; 40:3023-8.
8. Wang W, King MR. Multiscale modeling of platelet adhesion and thrombus growth. Annals of Biomedical Engineering 2012 Nov;40(11) 2345-54.

9. Tamagawa M, Kaneda H, Hiramoto M, Nagahama S. Simulation of thrombus formation in shear flows using Lattice Boltzmann Method. Artificial Organs 2009;33:604-10.

10. Zhao H, Shaqfeh E. SG. Numerical simulation of the margination of platelets in the microvasculature. Center of turbulence research, Stanford, Annual Research Briefs 2010.

11. Jordan A, David T, Homer-Vanniasinkam S, Graham A, Walker $P$. The effects of margination and red cell augmented platelet diffusivity on platelet adhesion in complex flow. Biorheology 2004;41:641-53.

12. Tokarev AA, Butylin AA, Ermakova EA, Shnol EE, Panasenko GP, Ataullakhanov FI. Finite platelet size could be responsible for platelet margination effect. Biophysical Journal 2011; 101:1835-43.

13. Zhao R, Kameneva MV, Antaki JF. Investigation of platelet margination phenomena at elevated shear stress. Biorheology 2007;44:161-77.

14. Mountrakis L, Hoekstra LE, Hoekstra AG. Where do the platelets go? A simulation study of fully resolved blood flow through aneurysmal vessels. Interface Focus 3: 20120089, Royal Society Publishing 2012.

15. Bhatnagar PL, Gross EP, Krook. A model for collision processes in gases. I. small amplitude processes in charged and neutral one-component systems. Physical Review 1954;94:511-25. 
16. Qian YH, D' Humières D, Lallemand P. Lattice BGK models for Navier-Stokes equation. Europhysics Letters 1992;17:479-84.

17. D'Humières D, Ginzburg I, Krafczyk M, Lallemand P, Luo LS. Multiple-relaxation-time lattice Boltzmann models in three dimensions. Philosophical Transactions of the Royal Society -
Series A: Mathematical, Physical and Engineering Sciences 2002;360:437-51.

18. Woldhuis B, Tangelder GJ, Slaaf DW, Reneman $R S$. Concentration profile of blood platelets differs in arterioles and venules. American Journal of Physiology 1992;262:H1217-H23.

\section{Gábor Závodszky}

Budapest University of Technology and Economics, Department of Hydrodynamic Systems H-1521 Budapest, Pf. 91.

Tel.: (+36) $1463-1111 / 5798$ 